\title{
Personalized TMS: role of RNA genotyping
}

\author{
Shawna Chan and Robert Bota \\ University of California Irvine, Irvine, California, USA
}

\begin{abstract}
Purpose - Noninvasive brain stimulation (NIBS) such a transcranial magnetic stimulation, intermittent theta burst stimulation, transcranial direct current stimulation and electroconvulsive therapy have emerged as an efficacious and well-tolerated therapy for treatment-resistant psychiatric disorders. While novel NIBS techniques are an exciting addition to the current repertoire of neuropsychiatric therapies, their success is somewhat limited by the wide range of treatment responses seen among treated patients.

Design/methodology/approach - In this study, the authors will review the studies on relevant genetic polymorphisms and discuss the role of RNA genotyping in personalizing NIBS.

Findings - Genome studies have revealed several genetic polymorphisms that may contribute for the heterogeneity of treatment response to NIBS where the presence of certain single nucleotide polymorphisms (SNPs) are associated with responders versus nonresponders.

Originality/value - Historically, mental illnesses have been arguably some of the most challenging disorders to study and to treat because of the degree of biological variability across affected individuals, the role of genetic and epigenetic modifications, the diversity of clinical symptomatology and presentations and the interplay with environmental factors. In lieu of these challenges, there has been a push for personalized medicine in psychiatry that aims to optimize treatment response based on one's unique characteristics.
\end{abstract}

Keywords Electroconvulsive therapy, Intermittent theta burst stimulation, Noninvasive brain stimulation, Transcranial direct current stimulation, Transcranial magnetic stimulation, Treatment-resistant psychiatric disorders

Paper type Research paper

\section{Introduction to noninvasive brain stimulation}

Noninvasive brain stimulation (NIBS) such a transcranial magnetic stimulation (TMS), intermittent theta burst stimulation (iTBS), transcranial direct current stimulation (tDCS) and electroconvulsive therapy (ECT) have emerged as an efficacious and well-tolerated therapy for treatment-resistant psychiatric disorders. These revolutionary neuromodulation techniques permit healthcare providers to alter cortical excitability without physically penetrating into brain tissue, such that sessions can be delivered in minutes on an outpatient basis, and patients may return to their daily activities on the same day. Additionally, these brain stimulation methods are postulated to induce changes on local neural activity that outlast the duration of stimulation and may be synergistically combined with pharmacotherapies to deliver therapeutic effects more quickly and with greater efficacy and durability than either modality alone (Rumi et al., 2005; Rossini et al., 2005; Liu et al., 2014; Chen et al., 2013; Bretlau et al., 2008).

\subsection{Transcranial magnetic stimulation (TMS)}

TMS therapy utilizes a computerized medical device to generate magnetic resonance imaging-strength (MRI-strength) magnetic fields that pass through the skull and induce focal electric currents that depolarize axons in target areas of brain tissue, modulating local neural activity with downstream effects

The current issue and full text archive of this journal is available on Emerald Insight at: www.emeraldinsight.com/2036-7465.htm

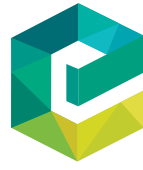

Mental Illness

11/2 (2019) 8-15

Emerald Publishing Limited [ISSN 2036-7465]

[DOI 10.1108/MIJ-10-2019-0004] in neural networks throughout the cortex (Lefaucheur et al., 2014; Allan et al., 2012). Daily TMS treatments are delivered over the course of 4-6weeks, and as they do not require anesthetic agents (as in ECT), patients are able to drive themselves to and from daily appointments. TMS was first recognized for its role in acute treatment of major depressive disorder (MDD) resistant to treatment with antidepressant medication or in patients unable to tolerate antidepressant medication. For the treatment of MDD, the strongest evidence exists for application of high-frequency TMS over the left dorsal lateral prefrontal cortex (DLPFC), and notably left DLPFC hypoactivity has been found on neuroimaging of patients with MDD (Baeken and De Raedt, 2011). The efficacy and safety of TMS have been demonstrated in various large, multisite randomized clinical trials, and it has been shown to produce clinically meaningful improvement in depressive symptoms as well significant improvement in quality of life; benefits were observed immediately following treatment with TMS and at follow-up 6-12 months later (O'Reardon et al., 2007; George et al., 2010; Levkovitz et al., 2015; Solvason et al., 2014). Safety considerations in the use of TMS include the development of headache or scalp discomfort at the site of application, hearing loss that may be prevented with

(C) Shawna Chan and Robert Bota. Published by Emerald Publishing Limited. This article is published under the Creative Commons Attribution (CC BY 4.0) licence. Anyone may reproduce, distribute, translate and create derivative works of this article (for both commercial and non-commercial purposes), subject to full attribution to the original publication and authors. The full terms of this licence may be seen at: http://creativecommons.org/licences/by/4.0/legalcode

Received 21 October 2019

Revised 21 October 2019

Accepted 21 October 2019 
hearing protection, a low incidence of seizures (lower than the risk reported for use of antidepressant medications) and vasovagal syncope (Janicak et al., 2008; Rossi et al., 2009; Tringali et al., 2012; Anderson et al., 2009).

Currently, TMS is approved by the Food and Drug Administration (FDA) for treatment of treatment-resistant major depression (O'Reardon et al., 2007; George et al., 2010; Levkovitz et al., 2015), pain associated with migraine headaches (Starling et al., 2018; Lipton et al., 2010; Bhola et al., 2015) and treatmentresistant obsessive-compulsive disorder (OCD) (Hawken et al., 2016). Investigations of its therapeutic role in various other neuropsychiatric disorders, including schizophrenia, bipolar disorder, Parkinson's disease, stroke-related deficits and chronic pain, are currently underway (Lefaucheur et al., 2014; He et al., 2017; Quan et al., 2015; Wobrock et al., 2015; Tavares et al., 2017; Hu et al., 2016; Cohen et al., 2018; Brys et al., 2016; Zheng et al., 2015; Ludemann-Podubecka et al., 2015; Attal et al., 2016; Sankarasubramanian et al., 2017).

\subsection{Intermittent theta burst stimulation (iTBS)}

iTBS is a more recent form of TMS that delivers $50 \mathrm{~Hz}$ bursts applied at $5 \mathrm{~Hz}$ that mimic endogenous hippocampal theta rhythms (Larson and Munkacsy, 2015). This approach has the advantage of delivering maximal synaptic long-term potentiation to hippocampal neurons, thereby enhancing the efficiency of neuromodulation by exerting longer-lasting effects on motor cortex excitability while requiring shorter treatment sessions ( 3 versus $38 \mathrm{~min}$ ) than conventional rTMS (Blumberger et al., 2018). The outcomes of iTBS have been shown to be equal to TMS for the treatment of MDD with a similar safety and tolerability profile where increased headache pain scores were reported with iTBS (Blumberger et al., 2018). Currently, iTBS is approved by the FDA for treatment-resistant MDD.

\subsection{Transcranial direct current stimulation (tDCS)}

tDCS is an investigational neuromodulation technique only available through research protocols that delivers a lowintensity current to specific cortical regions, typically the left DLPFC (Boggio et al., 2008). Instead of causing neuronal depolarization as in TMS, tDCS modulates spontaneous neuronal firing to modulate cortical excitatory tone (Nitsche et al., 2008). Anodal stimulation is associated with increased cortical excitability, while cathodal stimulation is associated with decreased cortical excitability, with effects potentially lasting beyond the period of stimulation (Tortella et al., 2015). For the treatment of depression, tDCS is theorized to modulate neural networks of cortical regions involved in mood regulation. Although some studies report that tDCS may be as effective as rTMS and antidepressant pharmacotherapy (Brunoni et al., 2016; Kalu et al., 2012; Berlim et al., 2013), notable side effects include the development of skin burns and the risk of switching from depression to hypomania (Loo et al., 2011; Loo et al., 2012; Brunoni et al., 2017).

\subsection{Personalized medicine: genomic data allow us to predict response for rapid treatment optimization for patients with refractory illnesses}

While novel NIBS techniques are an exciting addition to the current repertoire of neuropsychiatric therapies, their success is somewhat limited by the wide range of treatment responses seen among treated patients. Historically, mental illnesses have been arguably some of the most challenging disorders to study and to treat because of the degree of biological variability across affected individuals, the role of genetic and epigenetic modifications, the diversity of clinical symptomatology and presentations and the interplay with environmental factors. In lieu of these challenges, there has been a push for personalized medicine in psychiatry that aims to optimize treatment response based on one's unique characteristics. Genome studies have revealed several genetic polymorphisms that may contribute for the heterogeneity of treatment response to NIBS where the presence of certain SNPs are associated with responders versus non-responders. In this article, we will review the studies on relevant genetic polymorphisms and discuss the role of RNA genotyping in personalizing NIBS.

\section{Search strategy}

We searched PubMed, the primary biomedical database, between 2000 and October 2018, for literature relevant to topic of this review article. The search terms that were used included "transcranial magnetic stimulation," "transcranial direct stimulation," "theta burst stimulation," "polymorphism," "genomic" and "genotype." Bibliographies of articles were further hand-searched to identify additional relevant articles. Only published, peer-reviewed articles available in English were considered for this review.

\section{Significant genes and their polymorphisms}

\subsection{Brain-derived neurotrophic factor (Val66Met)}

Brain-derived neurotrophic factor (BDNF) gene encodes BDNF protein, which plays an important role in neuronal growth and differentiation, synaptic transmission, neuroprotection and neuroplasticity. BNDF binds tropomyosin receptor kinase B (TrkB) and activates signaling cascades, including the Ras/ MAPK-ERK, IRS-1/P13K/AKT and PLC/DAG/IP3 pathways, which activate downstream survival and growth genes (Bathina and Das, 2015). BDNF protein is highly expressed throughout various brain structures, including the hippocampus, basal forebrain, cortex and hypothalamus, and is implicated in learning, memory and higher cognitive function (Lu et al., 2014; Cunha et al., 2010). Deficits in BDNF signaling have been associated with predisposition toward neuropsychiatric disorders such as depression, bipolar disorder, Alzheimer's disease, Parkinson's disease and Huntington's disease (Binder and Scharfman, 2004). The BDNF Val66Met SNP is caused by a $\mathrm{G}>$ A point mutation at position 196, which destabilizes BDNF mRNA and interferes with trafficking and protein release (Wu et al., 2011). This polymorphism has been linked to hippocampal and cortical atrophy with the disruption of neural networks that traverse these areas. In the context of neuromodulation techniques that affect neural activity and excitatory tone at a local and network level, it is reasonable that the Val66Met SNP would influence treatment response.

BDNF is believed to be an important regulator rehabilitation-induced recovery following stroke by enhancing synaptic plasticity, increasing angiogenesis and neurogenesis and stimulating brain repair (Di Lazzaro et al., 2015; Berretta et al., 2014; Mirowska-Guzel et al., 2013). In stroke patients 
treated with TMS, the Val66Met polymorphism has been associated with poorer recovery of motor function compared to the homozygous ValVal genotype (Chang et al., 2014). The mechanism of how the Val66Met polymorphism interacts with NIBS in stroke rehabilitation remains to be found as inconsistent results are reported. In a study of 20 patients who had suffered from first-ever ischemic stroke, ValVal patients were found to have greater excitability over the unaffected hemisphere and increased inter-hemispheric imbalance compared to ValMet patients, and the difference became even more pronounced after application of iTBS; no difference in motor-evoked potential (MEP) threshold nor amplitude were noted between the two genotypes (Di Lazzaro et al., 2015). A study of 22 chronic stroke patients found that ValVal patients showed a significantly higher increase of MEP amplitude following treatment with high-frequency TMS over the ipsilesional M1 (Uhm et al., 2015); however, when evaluated in healthy volunteers and schizophrenic patients, the Val66Met polymorphism was not found to differentially influence MEP following TMS and tDCS, respectively (Hwang et al., 2015; Strube et al., 2014).

In patients suffering from major depression, Val66Met polymorphism appears to negatively impact treatment response to TMS. In a study of 36 patients with drugresistant depression, TMS treatment significantly improved depression symptoms as assessed by the Hamilton rating scale for depression (HAMD) in ValVal patients versus ValMet patients (Bocchio-Chiavetto et al., 2008). Additionally, in a study of 19 female patients with pharmacoresistant MDD treated with TMS, 80 per cent of patients who sustained significant HAMD score reduction at six months were of the ValVal genotype (Krstic et al., 2014). ValVal patients appear to show consistently better responses to TMS for MDD, and the mechanism may have to do with differences in modulation of motor cortex excitability and susceptibilities of synapses to undergo longterm potentiation (LTP) and long-term depression (LTD) (Cheeran et al., 2008; Cirillo et al., 2012). Interestingly, in a study of 40 healthy volunteers, the effect of BDNF polymorphism on cortical excitability following highfrequency TMS was only observed after delivery of subthreshold intensity ( 90 per cent of resting motor threshold (rMT)), but not supra-threshold intensity (110 per cent of rMT); no significant side effects were observed between the treatments. This finding suggests the mechanism may be even more complex, where BDNF polymorphism affects the threshold for likelihood of TMS-induced changes, with differences more likely to be observed when delivering weaker intensity. If this were the case, the treatment plan for patients with known Val66Met polymorphism may be safely adjusted to increase likelihood of treatment response to TMS. However, Val66Met polymorphism was not found to impact treatment response to tDCS (Brunoni et al., 2013).

\subsection{Dopamine D2 receptor (-957 C > T) and catechol-O- metyltransferase (Val158Met)}

The dopamine D2 receptor (DRD2) gene encodes a G-proteincoupled receptor that is highly expressed in the striatum and nucleus accumbens that mediates a variety of cognitive functions, including learning and memory, attention, reward behavior and pain response (Gluskin and Mickey, 2016). The most frequently studied DRD2 genetic variant is the Glu713Lys polymorphism caused by a missense $\mathrm{C}>\mathrm{T}$ mutation which appears to affect D2 receptor binding potential (Wagner et al., 2014; Savitz et al., 2013), receptor availability (Smith et al., 2017; Hirvonen et al., 2009) and mRNA stability and synthesis of the D2 receptor (Duan et al., 2003). Aberrant dopamine signaling is involved in the pathogenesis of various mental illnesses, including schizophrenia, bipolar disorder, Parkinson's disease, Huntington's disease and attention deficit disorder (Beaulieu and Gainetdinov, 2011), and dopamine receptors are frequently pharmacologic targets in the treatment of psychiatric conditions.

Because treatment with NIBS involves long-term modulation of cortical excitability, DRD2 variants are especially relevant in patients receiving NIBS because the D2 receptor has been shown to have dose-dependent effects on neuroplasticity. In healthy adults, D2 receptor blockade with sulpiride abolished tDCS-induced changes of excitability in both anodal and cathodal tDCS after-effects 5 min following stimulation (Nitsche et al., 2006). A similar effect was seen in healthy adult undergoing TBS, where administration of sulpiride blocked the excitatory effects of iTBS and inhibitory effects of continuous TBS (Monte-Silva et al., 2011), further supporting the important role of DRD2 in synaptic plasticity and NIBS outcomes.

For the treatment of neuropathic pain, rTMS of the motor cortex has been shown to produce analgesic effects via activation of the endogenous opioid network (de Andrade et al., 2011; Maarrawi et al., 2007). Meanwhile, DRD2 availability is known to be involved in pain modulatory capacity and response to pain (Pertovaara et al., 2004; Hagelberg et al., 2002); hence, activation of the striatal dopamine system may lead to release of endogenous opioids. In a study of healthy subjects and patients with neuropathic orofacial pain genotyped for the DRD2 gene $957 \mathrm{C}>\mathrm{T}$ polymorphism, healthy subjects homozygous for the DRD2 $957 \mathrm{~T}$ allele had increased thermal baseline sensitivity of facial skin measured by thermal detection threshold; additionally, rTMS to the sensorimotor cortex was only able to produce an analgesic effect in healthy 957TT homozygotes. Meanwhile, the prevalence of the 957TT allele was higher in patients with chronic neuropathic orofacial pain when compared with the general population, and patients with the 957TT allele experienced more severe pain symptoms. The authors suggest that DRD2 polymorphisms are not only implicated in pain sensitivity and susceptibility to chronic neuropathic pain but also in the analgesic efficacy of rTMS (Jaaskelainen et al., 2014). However, the clinical significance of DRD2 $957 \mathrm{C}>\mathrm{T}$ polymorphism remains unclear, as upon evaluation of clinical symptoms through Brief Pain Inventory scores, no correlation was found between genetic polymorphisms and patient-reported analgesia following treatment with rTMS in the same subset of chronic pain patients (Lindholm et al., 2015).

The catechol-O-methyltransferase (COMT) enzyme has been hypothesized to play a role in DRD2 variants because of its function in metabolizing and degrading dopamine. The COMT missense variant Val158Met modulates COMT activity and subsequently decreased dopamine levels in the DLPFC, where the Val/Val carriers demonstrate the most rapid dopamine metabolism, Val/Met carriers demonstrate 
intermediate dopamine metabolism and Met/Met carriers demonstrate the slowest metabolism (Chen et al., 2004). COMT activity is clinically relevant as higher enzymatic activity may lead to lower DLPFC dopamine signaling and impairment of cortical function. In a study of healthy adults, COMT Met/Met carriers were found to have lower set-shifting ability after anodal tDCS to DLPFC (Plewnia et al., 2013). Response inhibition, an important component of executive function, was found to be significantly impaired in healthy COMT Val/Val carriers after cathodal tDCS to DLPFC, while no effect of cathodal stimulation was seen in healthy Metcarriers (Nieratschker et al., 2015). The COMT genotype has also been found to interact with tDCS-linked working memory performance and tDCS intensity, where Val/Val homozygotes showed improved visual working memory and spatial working memory after receiving intermediate intensity anodal frontoparietal tDCS and low-intensity tDCS, respectively, while Met/Met homozygotes showed decreased spatial working memory performance following high-intensity tDCS (Stephens et al., 2017).

Interestingly, in patients with schizophrenia, homozygosity for COMT Val/Val is associated with significantly greater reduction of auditory hallucinations following tDCS to DLPFC compared to Val/Met and Met/Met carriers (Shivakumar et al., 2015; Chhabra et al., 2018). In the same cohort of patients, the authors also found that the neuregulin-1 (NRG1) rs35753505 polymorphism, which has been studied extensively for its potential role in the pathogenesis of schizophrenia, further decreased auditory hallucinations when present with COMT Val/Val. These findings suggest that the COMT Val158Met polymorphism influences the effect of tDCS on cognitive flexibility, which may ultimately lead to different levels of clinic benefit dependent on one's COMT genotype. Together, the DRD2 G > T and COMT Val158Met polymorphisms have been shown to affect important cognitive functions such as motor learning and performance, working memory and executive function (Klaus et al., 2017; Xu et al., 2007a; Noohi et al., 2014); their genetic effects may impact clinical outcomes in patients receiving NIBS and should be considered when determining optimal dosing and treatment regimens.

\subsection{5-HT1A (rs6295) and 5-HHT (SERTPR/5-HTTLPR)}

The serotonergic system regulates a diverse range of cognitive functions, including mood, sexual behavior, learning and memory. The role of serotonin, also known as 5-hydroxytryptamine (5-HT), has been widely studied in the pathogenesis of major depressive disorder in which the monoamine hypothesis of depression states that serotonin deficiency is linked with depression. Repetitive TMS to the DLPFC is currently an adjuvant treatment for treatmentresistant depression and theoretically enhances neuronal activity of serotonergic and/or dopaminergic systems. Animal studies have shown increased levels of 5-HT in the hippocampus and amygdala (Juckel et al., 1999), 5-HT receptor modulation throughout the brain (Gur et al., 2000; Ben-Shachar et al., 1999) and changes in 5-HT metabolism (Sibon et al., 2007) in limbic areas following TMS. Genetic polymorphisms that may affect antidepressant responses to TMS include: the short (s) and long (1) variants of the serotonin transporter promoter region (SERTPR/5HTTLPR), where the long variant has longer basal activity and may facilitate better response to selective serotonin reuptake inhibitors (SSRIs) (Serretti et al., 2007; Porcelli et al., 2012; Zanardi et al., 2001; Durham et al., 2004), and a $\mathrm{G}$ to $\mathrm{C}$ substitution at -1019 of the $5-\mathrm{HT} 1 \mathrm{~A}$ serotonergic receptor promoter region (rs6295), where C/C homozygotes demonstrated better response to SSRIs, while G/G homozygotes were over-represented in depressed patients when compared to controls (Hong et al., 2006; Arias et al., 2005; Lemonde et al., 2003).

Several studies have evaluated these polymorphisms in the context of NIBS for the treatment of depression. In a study in patients with a history of major depressive disorder, patients with the 1 allele of 5-HTT showed greater Hamilton Depression Rating Scale (HDRS) score reduction than s/s homozygotes, although the influence of the 5-HTT genotype was the same between active and sham TMS (Zanardi et al., 2007). The authors suggested that perhaps the 5-HTT genotype not only affects treatment response but also duration of symptoms; therefore, improvement of symptoms could also be observed in placebo treatment (Bocchio-Chiavetto et al., 2008). A separate study of patients with treatment-resistance depression also found greater HDRS score reduction in $1 / 1$ homozygotes following rTMS; however in this study, no influence of the 5-HTT polymorphism was seen following sham treatment. For the 5-HT1A polymorphism, studies disorder have shown that following rTMS to the left DLPFC, patients with the $\mathrm{C} / \mathrm{C}$ genotype had significantly greater improvement of depression as evaluated by the HDRS for depression than G/G and C/G individuals (Zanardi et al., 2007; Malaguti et al., 2011). Further studies are needed to clarify the utility of the 5-HTT and 5-HT1A polymorphisms to optimize NIBS outcomes for patients with treatment-resistance depression, and existing studies suggest these polymorphisms may be potential genetic predictors of treatment efficacy.

\subsection{TRPV1 (rs222747 and rs222749)}

The transient receptor potential vanillioid (TRPV1) gene encodes for nonselective cation channels that regulate glutamate release in response to a variety of stimuli such as endocannabinoids, eicosanoids, vanilloid compounds, voltage and heat (Suh and Oh, 2005; De Petrocellis and Marzo, 2005). TRPV1 has been shown to be important in synaptic plasticity, anxiety and fear and hippocampal long-term potentiation and may also be involved long-term depression (Marsch et al., 2007; Maione et al., 2009; Gibson et al., 2008). The "G" allele of rs222747 polymorphism leads to increased TRPV1 mRNA and protein expression on the cell surface as well as increased glutamate release in response, while rs222749 polymorphism does not appear to alter the functionality of the TRPV1 channel (Xu et al., 2007b). Following TMS to the right primary motor cortex, healthy adults with the G/G variant of rs222747 demonstrated significantly greater short-interval intracortical facilitation, which is believed to mirror the activation of glutamatergic cortical interneurons, while allelic variants of rs222749 did not show differences in cortical response to TMS (Mori et al., 2012). Hence, TRPV1 channels may also be implicated in cortical excitability with some polymorphisms facilitating greater cortical response to NIBS than others. 


\subsection{GRN}

Granulin (GRN) encodes progranulin, a secreted neuroregulatory growth factor believed to play a central role in brain development and neurodegeneration (van Swieten and Heutink, 2008; Petkau et al., 2012). Various mice and human studies have demonstrated that progranulin insufficiency is associated with familial frontotemporal dementia (FTD), where structural changes such as decreased brain volumes and dendritic density as well as reduced functional connectivity were observed prior to the onset of FTD symptoms (Borroni et al., 2008; Pievani et al., 2014; Dopper et al., 2014; Rohrer et al., 2015). In a study of presymptomatic GRN mutation carriers (g.1977_1980delCACT and IVS6 + 5_8delGTGA) and nonmutation carriers, TMS to the primary hand motor cortex demonstrated impaired shortinterval intracortical inhibition (SICI) and intracortical facilitation (ICF) of the right insula among GRN mutation carriers. Interestingly, brain MRI also noted increased cortical thickness in the supramarginal and superior parietal gyri and decreased surface area in the precuneus and inferior partial gyrus for GRN mutation carriers, which inversely correlated with SICI/ICF of the right insula (Gazzina et al., 2018) $\diamond \mathrm{GABA} /$ glutamatergic impairment.

\subsection{GRIN1 (rs4880213) and GRIN2B (rs1805247), SNCA (Ala53Thr), SCNA1, DAT1, GAG (rs11789969)}

\section{References}

Allan, C., et al. (2012), "Transcranial stimulation in depression", British Fournal of Psychiatry, Vol. 200 No. 1, pp. 10-11.

Anderson, B.S., et al. (2009), "Decreasing procedural pain over time of left prefrontal rTMS for depression: initial results from the open-label phase of a multi-site trial (OPTTMS)", Brain Stimulation, Vol. 2 No. 2, pp. 88-92.

Arias, B., et al. (2005), "Evidence for a combined genetic effect of the 5-HT(1A) receptor and serotonin transporter genes in the clinical outcome of major depressive patients treated with citalopram", fournal of Psychopharmacology, Vol. 19 No. 2, pp. 166-172.

Attal, N., et al. (2016), "Repetitive transcranial magnetic stimulation and transcranial direct-current stimulation in neuropathic pain due to radiculopathy: a randomized shamcontrolled comparative study", PAIN, Vol. 157 No. 6, pp. 1224-1231.

Baeken, C. and De Raedt, R. (2011), "Neurobiological mechanisms of repetitive transcranial magnetic stimulation on the underlying neurocircuitry in unipolar depression", Dialogues Clin Neurosci, Vol. 13 No. 1, pp. 139-145.

Bathina, S. and Das, U.N. (2015), "Brain-derived neurotrophic factor and its clinical implications", Archives of Medical Science, Vol. 11 No. 6, pp. 1164-1178.

Beaulieu, J.M. and Gainetdinov, R.R. (2011), "The physiology, signaling, and pharmacology of dopamine receptors", Pharmacological Reviews, Vol. 63 No. 1, pp. 182-217.

Ben-Shachar, D., et al. (1999), "Chronic repetitive transcranial magnetic stimulation alters beta-adrenergic and 5-HT2 receptor characteristics in rat brain", Brain Research, Vol. 816 No. 1, pp. 78-83.

Berlim, M.T., Van den Eynde, F. and Daskalakis, Z.J. (2013), "Clinical utility of transcranial direct current stimulation
(tDCS) for treating major depression: a systematic review and meta-analysis of randomized, double-blind and shamcontrolled trials", fournal of Psychiatric Research, Vol. 47 No. 1, pp. 1-7.

Berretta, A., Tzeng, Y.C. and Clarkson, A.N. (2014), "Poststroke recovery: the role of activity-dependent release of brain-derived neurotrophic factor", Expert Review of Neurotherapeutics, Vol. 14 No. 11, pp. 1335-1344.

Bhola, R., et al. (2015), "Single-pulse transcranial magnetic stimulation (sTMS) for the acute treatment of migraine: evaluation of outcome data for the UK post market pilot program", Fournal of Headache Pain, Vol. 16, p. 535.

Binder, D.K. and Scharfman, H.E. (2004), "Brain-derived neurotrophic factor”, Growth Factors, Vol. 22 No. 3, pp. 123-131.

Blumberger, D.M., et al. (2018), "Effectiveness of theta burst versus high-frequency repetitive transcranial magnetic stimulation in patients with depression (THREE-D): a randomised non-inferiority trial", The Lancet, Vol. 391 No. 10131, pp. 1683-1692.

Bocchio-Chiavetto, L., et al. (2008), "5-HTTLPR and BDNF Val66Met polymorphisms and response to rTMS treatment in drug resistant depression", Neuroscience Letters, Vol. 437 No. 2, pp. 130-134.

Boggio, P.S., et al. (2008), “A randomized, double-blind clinical trial on the efficacy of cortical direct current stimulation for the treatment of major depression", International fournal of Neuropsychopharmacology, Vol. 11 No. 2, pp. 249-254.

Borroni, B., et al. (2008), "Brain magnetic resonance imaging structural changes in a pedigree of asymptomatic progranulin mutation carriers", Rejuvenation Research, Vol. 11 No. 3, pp. 585-595.

Bretlau, L.G., et al. (2008), "Repetitive transcranial magnetic stimulation (rTMS) in combination with escitalopram in patients with treatment-resistant major depression: a doubleblind, randomised, sham-controlled trial", Pharmacopsychiatry, Vol. 41 No. 2, pp. 41-47.

Brunoni, A.R., et al. (2013), "Impact of 5-HTTLPR and BDNF polymorphisms on response to sertraline versus transcranial direct current stimulation: implications for the serotonergic system", European Neuropsychopharmacology, Vol. 23 No. 11, pp. 1530-1540.

Brunoni, A.R., et al. (2016), "Transcranial direct current stimulation for acute major depressive episodes: metaanalysis of individual patient data", British fournal of Psychiatry, Vol. 208 No. 6, pp. 522-531.

Brunoni, A.R., et al. (2017), "Trial of electrical Direct-Current therapy versus escitalopram for depression", New England fournal of Medicine, Vol. 376 No. 26, pp. 2523-2533.

Brys, M., et al. (2016), "Multifocal repetitive TMS for motor and mood symptoms of Parkinson disease: a randomized trial”, Neurology, Vol. 87 No. 18, pp. 1907-1915.

Chang, W.H., et al. (2014), "BDNF polymorphism and differential rTMS effects on motor recovery of stroke patients", Brain Stimulation, Vol. 7 No. 4, pp. 553-558.

Cheeran, B., et al. (2008), "A common polymorphism in the brain-derived neurotrophic factor gene (BDNF) modulates human cortical plasticity and the response to rTMS", The Fournal of Physiology, Vol. 586 No. 23, pp. 5717-5725. 
Chen, J., et al. (2004), "Functional analysis of genetic variation in catechol-O-methyltransferase (COMT): effects on mRNA, protein, and enzyme activity in postmortem human brain", The American fournal of Human Genetics, Vol. 75 No. 5, pp. 807-821.

Chen, S.J., et al. (2013), "Superior antidepressant effect occurring 1 month after rTMS: add-on rTMS for subjects with medication-resistant depression", Neuropsychiatric Disease and Treatment, Vol. 9 No. 9, pp. 397-401.

Chhabra, H., et al. (2018), "Gene polymorphisms and response to transcranial direct current stimulation for auditory verbal hallucinations in schizophrenia", Acta Neuropsychiatrica, Vol. 30 No. 4, pp. 218-225.

Cirillo, J., et al. (2012), "Differential modulation of motor cortex excitability in BDNF met allele carriers following experimentally induced and use-dependent plasticity", European fournal of Neuroscience, Vol. 36 No. 5, pp. 2640-2649.

Cohen, O.S., et al. (2018), "Repetitive deep TMS for Parkinson disease: a 3-month double-blind, randomized sham-controlled study", fournal of Clinical Neurophysiology, Vol. 35 No. 2, pp. 159-165.

Cunha, C., Brambilla, R. and Thomas, K.L. (2010), "A simple role for BDNF in learning and memory?", Frontiers in Molecular Neuroscience, Vol. 3, pp. 1.

de Andrade, D.C., et al. (2011), "Neuropharmacological basis of rTMS-induced analgesia: the role of endogenous opioids", Pain, Vol. 152 No. 2, pp. 320-326.

De Petrocellis, L. and Marzo, V.D. (2005), "Lipids as regulators of the activity of transient receptor potential type V1 (TRPV1) channels", Life Sciences, Vol. 77 No. 14, pp. 1651-1666.

Di Lazzaro, V., et al. (2015), "Val66Met BDNF gene polymorphism influences human motor cortex plasticity in acute stroke", Brain Stimulation, Vol. 8 No. 1, pp. 92-96.

Dopper, E.G., et al. (2014), "Structural and functional brain connectivity in presymptomatic familial frontotemporal dementia", Neurology, Vol. 83 No. 2, pp. e19-26.

Duan, J., et al. (2003), "Synonymous mutations in the human dopamine receptor D2 (DRD2) affect mRNA stability and synthesis of the receptor", Human Molecular Genetics, Vol. 12 No. 3, pp. 205-216.

Durham, L.K., et al. (2004), "The serotonin transporter polymorphism, 5HTTLPR, is associated with a faster response time to sertraline in an elderly population with major depressive disorder", Psychopharmacology (Psychopharmacology), Vol. 174 No. 4, pp. 525-529.

Gazzina, S., et al. (2018), "Neuroanatomical correlates of transcranial magnetic stimulation in presymptomatic granulin mutation carriers", Brain Topography, Vol. 31 No. 3, pp. 488-497.

George, M.S., et al. (2010), "Daily left prefrontal transcranial magnetic stimulation therapy for major depressive disorder: a sham-controlled randomized trial", Archives of General Psychiatry, Vol. 67 No. 5, pp. 507-516.

Gibson, H.E., et al. (2008), "TRPV1 channels mediate longterm depression at synapses on hippocampal interneurons", Neuron, Vol. 57 No. 5, pp. 746-759.

Gluskin, B.S. and Mickey, B.J. (2016), "Genetic variation and dopamine D2 receptor availability: a systematic review and meta-analysis of human in vivo molecular imaging studies", Translational Psychiatry, Vol. 6 No. 3, p. e747.

Gur, E., et al. (2000), "Chronic repetitive transcranial magnetic stimulation induces subsensitivity of presynaptic serotonergic autoreceptor activity in rat brain", NeuroReport, Vol. 11 No. 13, pp. 2925-2929.

Hagelberg, N., et al. (2002), "Dopamine D2 receptor binding in the human brain is associated with the response to painful stimulation and pain modulatory capacity", Pain, Vol. 99 No. 1, pp. 273-279.

Hawken, E.R., et al. (2016), "Transcranial magnetic stimulation of the supplementary motor area in the treatment of obsessive-compulsive disorder: a multi-site study", International fournal of Molecular Sciences, Vol. 17 No. 3, p. 420.

He, H., et al. (2017), "Repetitive transcranial magnetic stimulation for treating the symptoms of schizophrenia: a PRISMA compliant meta-analysis", Clinical Neurophysiology, Vol. 128 No. 5, pp. 716-724.

Hirvonen, M.M., et al. (2009), "C957T polymorphism of the human dopamine D2 receptor gene predicts extrastriatal dopamine receptor availability in vivo", Progress in NeuroPsychopharmacology and Biological Psychiatry, Vol. 33 No. 4, pp. 630-636.

Hong, C.J., et al. (2006), "Response to fluoxetine and serotonin 1A receptor (C-1019G) polymorphism in Taiwan Chinese major depressive disorder", The Pharmacogenomics fournal, Vol. 6 No. 1, pp. 27-33.

$\mathrm{Hu}$, S.H., et al. (2016), "Efficacy of repetitive transcranial magnetic stimulation with quetiapine in treating bipolar II depression: a randomized, double-blinded, control study", Scientific Reports, Vol. 6 No. 1, p. 30537.

Hwang, J.M., et al. (2015), "Different responses to facilitatory rTMS according to BDNF genotype", Clinical Neurophysiology, Vol. 126 No. 7, pp. 1348-1353.

Jaaskelainen, S.K., et al. (2014), "Variation in the dopamine D2 receptor gene plays a key role in human pain and its modulation by transcranial magnetic stimulation", Pain, Vol. 155 No. 10, pp. 2180-2187.

Janicak, P.G., et al. (2008), "Transcranial magnetic stimulation in the treatment of major depressive disorder: a comprehensive summary of safety experience from acute exposure, extended exposure, and during reintroduction treatment", The fournal of Clinical Psychiatry, Vol. 69 No. 2, pp. 222-232.

Juckel, G., Mendlin, A. and Jacobs, B.L. (1999), "Electrical stimulation of rat medial prefrontal cortex enhances forebrain serotonin output: implications for electroconvulsive therapy and transcranial magnetic stimulation in depression", Neuropsychopharmacology, Vol. 21 No. 3, pp. 391-398.

Kalu, U.G., et al. (2012), "Transcranial direct current stimulation in the treatment of major depression: a metaanalysis", Psychological Medicine, Vol. 42 No. 9, pp. 1791-1800.

Klaus, K., et al. (2017), "The effect of COMT Val158Met and DRD2 C957T polymorphisms on executive function and the impact of early life stress", Brain and Behavior, Vol. 7 No. 5, p. e00695.

Krstic, J., et al. (2014), "Low-frequency repetitive transcranial magnetic stimulation in the right prefrontal cortex combined 
with partial sleep deprivation in treatment-resistant depression: a randomized sham-controlled trial”, $\mathcal{F}$ Ect, Vol. 30 No. 4, pp. 325-331.

Larson, J. and Munkacsy, E. (2015), "Theta-burst LTP", Brain Research, Vol. 1621, pp. 38-50.

Lefaucheur, J.P., et al. (2014), "Evidence-based guidelines on the therapeutic use of repetitive transcranial magnetic stimulation (rTMS)", Clinical Neurophysiology, Vol. 125 No. 11, pp. 2150-2206.

Lemonde, S., et al. (2003), "Impaired repression at a 5hydroxytryptamine $1 \mathrm{~A}$ receptor gene polymorphism associated with major depression and suicide", The fournal of Neuroscience, Vol. 23 No. 25, pp. 8788-8799.

Levkovitz, Y., et al. (2015), "Efficacy and safety of deep transcranial magnetic stimulation for major depression: a prospective multicenter randomized controlled trial", World Psychiatry, Vol. 14 No. 1, pp. 64-73.

Lindholm, P., et al. (2015), "Right secondary somatosensory cortex-a promising novel target for the treatment of drugresistant neuropathic orofacial pain with repetitive transcranial magnetic stimulation", PAIN, Vol. 156 No. 7, pp. 1276-1283.

Lipton, R.B., et al. (2010), "Single-pulse transcranial magnetic stimulation for acute treatment of migraine with aura: a randomised, double-blind, parallel-group, sham-controlled trial”, The Lancet Neurology, Vol. 9 No. 4, pp. 373-380.

Liu, B., et al. (2014), "Repetitive transcranial magnetic stimulation as an augmentative strategy for treatment-resistant depression, a Meta-analysis of randomized, double-blind and shamcontrolled study", BMC Psychiatry, Vol. 14 No. 1, pp. 342.

Loo, C.K., et al. (2011), "Avoiding skin burns with transcranial direct current stimulation: preliminary considerations", The International fournal of Neuropsychopharmacology, Vol. 14 No. 3, pp. 425-426.

Loo, C.K., et al. (2012), "Transcranial direct current stimulation for depression: 3-week, randomised, sham-controlled trial”, British Fournal of Psychiatry, Vol. 200 No. 1, pp. 52-59.

Lu, B., Nagappan, G. and Lu, Y. (2014), "BDNF and synaptic plasticity, cognitive function, and dysfunction", Handb Exp Pharmacol, Vol. 220, pp. 223-250.

Ludemann-Podubecka, J., Bosl, K. and Nowak, D.A. (2015), "Repetitive transcranial magnetic stimulation for motor recovery of the upper limb after stroke", Progress in Brain Research, Vol. 218, pp. 281-311.

Maarrawi, J., et al. (2007), "Motor cortex stimulation for pain control induces changes in the endogenous opioid system", Neurology, Vol. 69 No. 9, pp. 827-834.

Maione, S., et al. (2009), "TRPV1 channels control synaptic plasticity in the developing superior colliculus", The fournal of Physiology, Vol. 587 No. 11, pp. 2521-2535.

Malaguti, A., et al. (2011), "Role of COMT, 5-HT(1A), and SERT genetic polymorphisms on antidepressant response to transcranial magnetic stimulation", Depression and Anxiety, Vol. 28 No. 7, pp. 568-573.

Marsch, R., et al. (2007), "Reduced anxiety, conditioned fear, and hippocampal long-term potentiation in transient receptor potential vanilloid type 1 receptor-deficient mice", Fournal of Neuroscience, Vol. 27 No. 4, pp. 832-839.

Mirowska-Guzel, D., et al. (2013), "Association between BDNF-196 G>a and BDNF-270 $\mathrm{C}>\mathrm{T}$ polymorphisms,
BDNF concentration, and rTMS-supported long-term rehabilitation outcome after ischemic stroke", NeuroRehabilitation, Vol. 32 No. 3, pp. 573-582.

Monte-Silva, K., et al. (2011), "D2 receptor block abolishes theta burst stimulation-induced neuroplasticity in the human motor cortex", Neuropsychopharmacology, Vol. 36 No. 10, pp. 2097-2102.

Mori, F., et al. (2012), "TRPV1 channels regulate cortical excitability in humans", fournal of Neuroscience, Vol. 32 No. 3, pp. 873-879.

Nieratschker, V., et al. (2015), "The COMT val/met polymorphism modulates effects of tDCS on response inhibition", Brain Stimulation, Vol. 8 No. 2, pp. 283-288.

Nitsche, M.A., et al. (2006), "Dopaminergic modulation of long-lasting direct current-induced cortical excitability changes in the human motor cortex", European fournal of Neuroscience, Vol. 23 No. 6, pp. 1651-1657.

Nitsche, M.A., et al. (2008), "Transcranial direct current stimulation: state of the art 2008", Brain Stimulation, Vol. 1 No. 3, pp. 206-223.

Noohi, F., et al. (2014), "Association of COMT val158met and DRD2 G>T genetic polymorphisms with individual differences in motor learning and performance in female young adults", fournal of Neurophysiology, Vol. 111 No. 3, pp. 628-640.

O'Reardon, J.P., et al. (2007), "Efficacy and safety of transcranial magnetic stimulation in the acute treatment of major depression: a multisite randomized controlled trial", Biological Psychiatry, Vol. 62 No. 11, pp. 1208-1216.

Pertovaara, A., et al. (2004), "Striatal dopamine D2/D3 receptor availability correlates with individual response characteristics to pain", European Fournal of Neuroscience, Vol. 20 No. 6, pp. 1587-1592.

Petkau, T.L., et al. (2012), "Synaptic dysfunction in progranulin-deficient mice", Neurobiology of Disease, Vol. 45 No. 2, pp. 711-722.

Pievani, M., et al. (2014), "Pattern of structural and functional brain abnormalities in asymptomatic granulin mutation carriers", Alzheimer's \& Dementia, Vol. 10 No. 5, pp. S354-S363 e1.

Plewnia, C., et al. (2013), "Effects of transcranial direct current stimulation ( $\mathrm{tDCS}$ ) on executive functions: influence of COMT val/met polymorphism", Cortex, Vol. 49 No. 7, pp. 1801-1807.

Porcelli, S., Fabbri, C. and Serretti, A. (2012), "Meta-analysis of serotonin transporter gene promoter polymorphism (5HTTLPR) association with antidepressant efficacy", European Neuropsychopharmacology, Vol. 22 No. 4, pp. 239-258.

Quan, W.X., et al. (2015), "The effects of high-frequency repetitive transcranial magnetic stimulation (rTMS) on negative symptoms of schizophrenia and the follow-up study", Neuroscience Letters, Vol. 584, pp. 197-201.

Rohrer, J.D., et al. (2015), "Presymptomatic cognitive and neuroanatomical changes in genetic frontotemporal dementia in the genetic frontotemporal dementia initiative (GENFI) study: a cross-sectional analysis", The Lancet Neurology, Vol. 14 No. 3, pp. 253-262.

Rossi, S., et al. (2009), "Safety, ethical considerations, and application guidelines for the use of transcranial magnetic stimulation in clinical practice and research", Clinical Neurophysiology, Vol. 120 No. 12, pp. 2008-2039. 
Rossini, D., et al. (2005), "Does rTMS hasten the response to escitalopram, sertraline, or venlafaxine in patients with major depressive disorder? A double-blind, randomized, shamcontrolled trial", The fournal of Clinical Psychiatry, Vol. 66 No. 12, pp. 1569-1575.

Rumi, D.O., et al. (2005), "Transcranial magnetic stimulation accelerates the antidepressant effect of amitriptyline in severe depression: a double-blind placebo-controlled study", Biological Psychiatry, Vol. 57 No. 2, pp. 162-166.

Sankarasubramanian, V., et al. (2017), "Transcranial direct current stimulation targeting primary motor versus dorsolateral prefrontal cortices: proof-of-Concept study investigating functional connectivity of thalamocortical networks specific to Sensory-Affective information processing", Brain Connectivity, Vol. 7 No. 3, pp. 182-196.

Savitz, J., et al. (2013), "DRD2/ANKK1 Taq1A polymorphism (rs1800497) has opposing effects on D2/3 receptor binding in healthy controls and patients with major depressive disorder", International fournal of Neuropsychopharmacology, Vol. 16 No. 9, pp. 2095-2101.

Serretti, A., et al. (2007), "Meta-analysis of serotonin transporter gene promoter polymorphism (5-HTTLPR) association with selective serotonin reuptake inhibitor efficacy in depressed patients", Molecular Psychiatry, Vol. 12 No. 3, pp. 247-257.

Shivakumar, V., et al. (2015), "Effect of tDCS on auditory hallucinations in schizophrenia: influence of catechol-Omethyltransferase (COMT) Val158Met polymorphism", Asian fournal of Psychiatry, Vol. 16, pp. 75-77.

Sibon, I., et al. (2007), "Acute prefrontal cortex TMS in healthy volunteers: effects on brain 11C-alphaMtrp trapping", NeuroImage, Vol. 34 No. 4, pp. 1658-1664.

Smith, C.T., et al. (2017), "The impact of common dopamine $\mathrm{D} 2$ receptor gene polymorphisms on $\mathrm{D} 2 / 3$ receptor availability: c $957 \mathrm{~T}$ as a key determinant in putamen and ventral striatum", Translational Psychiatry, Vol. 7 No. 4, pp. e1091.

Solvason, H.B., et al. (2014), "Improvement in quality of life with left prefrontal transcranial magnetic stimulation in patients with pharmacoresistant major depression: acute and six month outcomes", Brain Stimulation, Vol. 7 No. 2, pp. 219-225.

Starling, A.J., et al. (2018), “A multicenter, prospective, single arm, open label, observational study of sTMS for migraine prevention (ESPOUSE study)", Cephalalgia, Vol. 38 No. 6, pp. 1038-1048.

Stephens, J.A., Jones, K.T. and Berryhill, M.E. (2017), “Task demands, tDCS intensity, and the COMT val(158)met polymorphism impact tDCS-linked working memory training gains", Scientific Reports, Vol. 7 No. 1, pp. 13463.

Strube, W., et al. (2014), "BDNF-Val66Met-polymorphism impact on cortical plasticity in schizophrenia patients: a proof-of-concept study", International fournal of Neuropsychopharmacol, Vol. 18 No. 4.

Suh, Y.G. and Oh, U. (2005), "Activation and activators of TRPV1 and their pharmaceutical implication", Current Pharmaceutical Design, Vol. 11 No. 21, pp. 2687-2698.
Tavares, D.F., et al. (2017), "Treatment of bipolar depression with deep TMS: results from a Double-Blind, randomized, parallel group, sham-controlled clinical trial", Neuropsychopharmacology, Vol. 42 No. 13, pp. 2593-2601.

Tortella, G., et al. (2015), "Transcranial direct current stimulation in psychiatric disorders", World fournal of Psychiatry, Vol. 5 No. 1, pp. 88-102.

Tringali, S., et al. (2012), "Repetitive transcranial magnetic stimulation: hearing safety considerations", Brain Stimulation, Vol. 5 No. 3, pp. 354-363.

Uhm, K.E., et al. (2015), "BDNF genotype influence the efficacy of rTMS in stroke patients", Neuroscience Letters, Vol. 594, pp. 117-121.

van Swieten, J.C. and Heutink, P. (2008), "Mutations in progranulin (GRN) within the spectrum of clinical and pathological phenotypes of frontotemporal dementia", The Lancet Neurology, Vol. 7 No. 10, pp. 965-974.

Wagner, A.K., et al. (2014), "The influence of genetic variants on striatal dopamine transporter and D2 receptor binding after TBI", Fournal of Cerebral Blood Flow \& Metabolism, Vol. 34 No. 8, pp. 1328-1339.

Wobrock, T., et al. (2015), "Left prefrontal high-frequency repetitive transcranial magnetic stimulation for the treatment of schizophrenia with predominant negative symptoms: a sham-controlled, randomized multicenter trial", Biological Psychiatry, Vol. 77 No. 11, pp. 979-988.

Wu, Y.C., et al. (2011), "Dendritic trafficking of brain-derived neurotrophic factor mRNA: regulation by Translindependent and -independent mechanisms", fournal of Neurochemistry, Vol. 116 No. 6, pp. 1112-1121.

$\mathrm{Xu}, \mathrm{H}$., et al. (2007a), "DRD2 C957T polymorphism interacts with the COMT Val158Met polymorphism in human working memory ability", Schizophrenia Research, Vol. 90 Nos 1/3, pp. 104-107.

$\mathrm{Xu}, \mathrm{H}$., et al. (2007b), "Functional effects of nonsynonymous polymorphisms in the human TRPV1 gene", American fournal of Physiology-Renal Physiology, Vol. 293 No. 6, pp. F1865-76.

Zanardi, R., et al. (2001), "Factors affecting fluvoxamine antidepressant activity: influence of pindolol and 5HTTLPR in delusional and nondelusional depression", Biological Psychiatry, Vol. 50 No. 5, pp. 323-330.

Zanardi, R., et al. (2007), "Role of serotonergic gene polymorphisms on response to transcranial magnetic stimulation in depression", European Neuropsychopharmacology, Vol. 17 No. 10, pp. 651-657.

Zheng, C.J., Liao, W.J. and Xia, W.G. (2015), "Effect of combined low-frequency repetitive transcranial magnetic stimulation and virtual reality training on upper limb function in subacute stroke: a double-blind randomized controlled trail", Fournal of Huazhong University of Science and Technology [Medical Sciences]), Vol. 35 No. 2, pp. 248-254.

\section{Corresponding author}

Shawna Chan can be contacted at: shawnatc@hc.uci.edu 